\title{
Indication of the Coronavirus Model Using a Nanowire Biosensor ${ }^{\dagger}$
}

\author{
Vladimir Generalov ${ }^{1, *}$, Olga Naumova ${ }^{2}$, Dmitry Shcherbakov ${ }^{1}$, Alexander Safatov ${ }^{1}$, Boris \\ Zaitsev ${ }^{1}$, Elza Zaitseva 2, Galina Buryak ${ }^{1}$, Dmitry Shcheglov ${ }^{2}$, Anastasiy Cheremiskina ${ }^{1}$, Julia \\ Merkuryeva ${ }^{1}$ and Alexander Aseyev ${ }^{2}$ \\ 1 Federal State Research Institution State Research Center of Virology and Biotechnology “Vector”, 630559 \\ Koltsovo, Russia; scherbakov_dn@vector.nsc.ru (D.S.); safatov@vector.nsc.ru (A.S.); zaitsev@vector.nsc.ru \\ (B.Z.); buryak@vector.nsc.ru (G.B.); cheremiskina_aa@vector.nsc.ru (A.C.); j.a.merkulyeva@gmail.com (J.M.) \\ 2 Rzhanov Institute of Semiconductor Physics, Siberian Branch of the Russian Academy of Sciences, 630090 \\ Novosibirsk, Russia; naumova@isp.nsc.ru (O.N.); x-treme2008@yandex.ru (E.Z.); goldfinher@mail.ru (D.S.); \\ al.aseev2011@yandex.ru (A.A.) \\ * Correspondence: general@vector.nsc.ru; Tel.: +7-913-452-6073 \\ + Presented at the 1st International Electronic Conference on Biosensors, 2-17 November 2020; Available \\ online: https://iecb2020.sciforum.net/.
}

Received: date; Accepted: date; Published: date

\begin{abstract}
The presented results indicate virus like particles of the coronavirus (CVP) using a nanowire (NW) biosensor based on technology silicon on insulator. In the experiment, we used suspensions: CVP and of specific antibodies to the virus. Measurements of the current value of the field-effect transistor before and after the introduction of the CVP on the surface of the nanowire were performed. Results: - antibody + CVP complexes on the phase section with the surface of the nanowire modulate the current of the field-effect transistor; -CVP has an electrically positive charge on the phase section "nanowire surface-viral suspension»; - antibody + CVP complexes have an electrically negative charge on the phase section "nanowire surface-viral suspension"; -the sensitivity of the biosensor is made up of $10^{-18} \mathrm{M}$; - time display made up of 200-300 s.
\end{abstract}

Keywords: biosensor; silicon on insulator (CNI), nanowire; indication; virus; coronavirus; indication

\section{Introduction}

In early 2020, the world health organization announced an outbreak of coronavirus infection 2019-nCoV, which was first reported on 31 December 2019 in Wuhan, China, but on 11 March, given the rapid increase in the number of cases worldwide, it was characterized by the COVID-19 coronavirus pandemic [1]. The COVID-19 pandemic is accompanied by profound changes in everyday life, countries are experiencing economic downturns, and many traditional social, economic, and public health systems are under unprecedented strain. The health and social protection systems were shocked. The measures taken to control transmission of the virus have had extensive and profound socio-economic consequences [2].

Coronaviruses (Cov) are a large family of RNA - containing viruses that cause illness from the common cold to more serious diseases such as middle East respiratory syndrome (MERS-Cov) and severe acute respiratory syndrome (SARS-Cov). A new coronavirus (nCoV) is a strain not previously detected in humans [3]. Researchers note the most characteristic symptoms of the disease: fatigue, shortness of breath, headaches, fever, kidney failure, dry cough, bronchitis, but sometimes register specific pneumonia of the lungs, including with a fatal outcome. Currently, information about the clinic, prevention and treatment of this disease is still limited [3-5]. At the time of writing, there are 
no WHO-recommended medicines or vaccines designed to prevent or treat infection caused by the new coronavirus (2019-nCoV). At the same time, both in Russia and abroad, vaccines against coronavirus are being developed [6]. Other factors of containment of the coronavirus that the WHO notes are laboratory diagnostics. The most common method of laboratory diagnostics $2019-\mathrm{nCoV}$, is polymerase chain reaction (PCR). For her, the main type of biomaterial is a smear from the nasopharynx and/or mouth. In Russia, diagnostics are performed using test systems that have a Roszdravnadzor registration certificate and are approved for use in laboratories in the Russian Federation [6]. The method of PCR analysis gives a direct indication of the presence of a specific section of the pathogen's DNA in the material taken from the patient. Another effective method of ELISA diagnostic is based on specific binding of antigens and antibodies in the sample with further detection of their enzyme label [7].

The development of new more sensitive and rapid methods of virus indication and the study of the fundamental properties of virus-like coronavirus particles is an urgent problem in the treatment of epidemics and pandemics. The aim of this work is to indicate the virus of similar coronavirus particles using a KNI-NP transistor and study its properties as a particle.

\section{Materials and Methods}

The following materials and equipment were used in the research.

N-channel silicon nanowire field-effect transistors (Si-NW FET) were used as biosensors [8]. Schematic and optical images of the n-channel SI-NW FET biosensor obtained using an atomic force microscope (AFM) are shown Figure 1a-c. The thickness of the silicon layer ( $\mathrm{Si}$ ) was $28-30 \mathrm{~nm}$, the thickness of the hidden dielectric (BOX, buried oxide) was $200 \mathrm{~nm}$. The channel width of the sensor element of the transistor located between the contact areas of the source $S$ and the drain $D$ was $W=3$ microns, the length $\mathrm{L}=10$ microns Figure 1 a. During measurements, the voltage between the drain and the source of the transistor was $\mathrm{Vds}=0.15 \mathrm{~V}$. The structures BG gate was used as the second controlling electrode. It provided modulation of the electronic channel conductivity and selection of the transistor operating point mode in the Ids sub-threshold region (Vbg). A grounded reference platinum Pt electrode (RE) was inserted directly before measurements into a drop of viral suspension, which removed the possibility of an undesirable induced constant electric charge of the viral suspension.

a

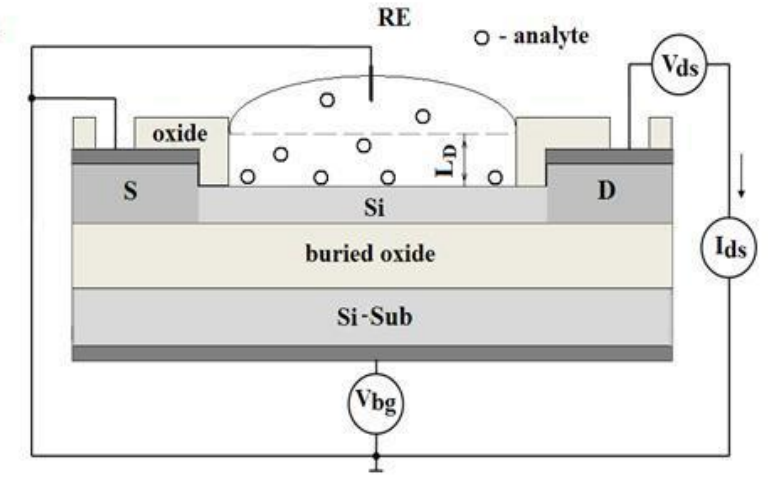

b

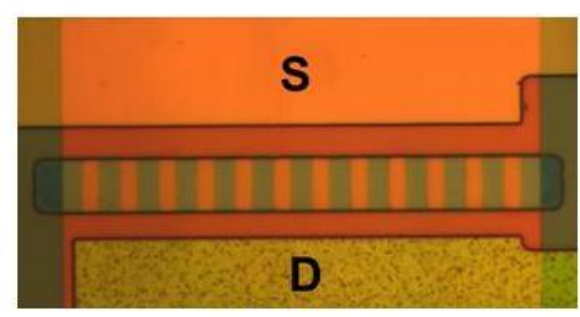

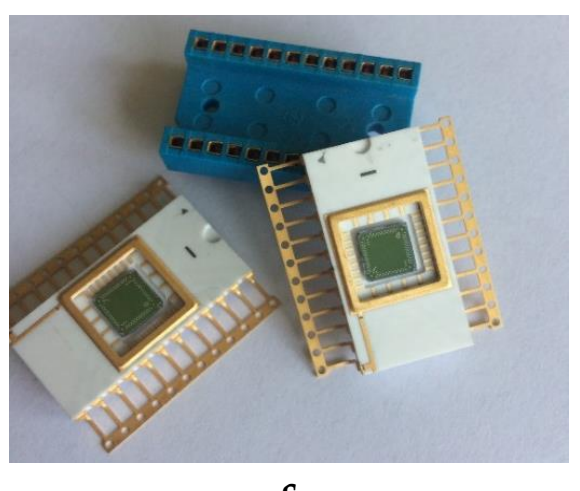

C 
Figure 1. (a)-Schematic image of the n-channel biosensor. o-suspension of antibody and virus-like coronavirus particles above the surface of the nanowire. RE-platinum reference electrode; $(\mathbf{b}, \mathbf{c})$-Optical image of a multichannel sensor on a chip between the contact areas of the source (S) and the drain (D).

The experiments used CVP at based on vesicular stomatitis virus that are not capable of replication. The CVP are modified by the s surface protein of the SARS-CoV-2 coronavirus. The remaining virion-forming proteins are represented by vesicular stomatitis virus proteins and genomic RNA defective in the g VSV coding region. The initial concentration of HPV IN the viral suspension was $10^{10}$ particles $/ \mathrm{mL}$. In experimental samples the amount of HPV was varied in the range from $10^{-18}$ to $10^{-13} \mathrm{M}$.

Specific antibodies to CVP were obtained based on the m396 antibody interacting with SARS [9]. To increase the affinity of antibodies to SARS-CoV-2, its structure was changed, L (leucine) in position 54 was replaced by $\mathrm{V}$ (valine).

Immediately before the experiment, the initial suspensions of antibodies and HPV were diluted with distilled water. Dilution was carried out, among other things, in order to create a suspension that does not conduct electric current. The high conductivity of the suspensions causes the transistors to be inoperable. All the biological material used in the work and samples based on them were prepared in the Federal state research center of Virology and biotechnology "vector" of Rospotrebnadzor.

Ethanol 96\%, Kemerovo pharmaceutical factory, Russia,

(3-Aminopropyl) triethoxysilane (APTES), Sigma-Aldrich, USA,

$25 \%$ ethanol solution of APTES.

Immediately before the experiment, the initial suspensions of antibodies and CVP were diluted with a distilled water one hundred times. Dilution was carried out in order to create a non -conducting electric current suspensions. The high conductivity of suspensions makes transistors inoperable.

Automatic pipette 1-2 mL and 10-100 mL, Lenpipet, Russia.

Atomic force microscope AFM SOLVER P47BIO NT-MDT, Russia.

The surface of the Si-NW FET was divided into two parts for to obtain two independent indicator transistors. The first transistor was called experimental, the second control. Sample preparation of the first a experimental transistor was carried out in the following technological cycle.

1. The procedure for cleaning the surface of the Si-NW FET was carried out as follows:

- $\quad 96 \%$ ethanol solution with a volume of $\mathrm{V}=10 \mu \mathrm{L}$ was applied and removed with a pipette to the surface of the SI-NW FET 20-30 times, then the surface was washed with distilled water according to the same scheme. The procedure was repeated 3 times. After it SI-NW FET was total drying.

2. The cleaned and dried surface of the SI-NW FET was modified by $25 \%$ ethanol solution APTES with a volume of V =5-10 $\mu \mathrm{L}$. The SI-NW FET was placed in a Petri dish and covered with a lid. A $25 \%$ ethanol solution of APTES was previously added to the Petri dish to create the vapors of the specified analyte. The SI-NW FET was in the specified pairs for $5 \mathrm{~h}$, at $\mathrm{T}=20^{\circ} \mathrm{C}$. The fill level of the analyte did not exceed the surface height of the SI-NW FET.

3. After $5 \mathrm{~h}$, the surface of the SI-NW FET was washed by distilled water. The procedure was repeated 3 times. The after it SI-NW FET was total drying.

4. A suspension of diluted antibodies with a volume of $\mathrm{V}=5-10 \mu \mathrm{L}$ was applied to the dry surface of the SI-NW FET. Then the SI-NW FET was placed in a Petri dish with water. The cup was covered with a lid and placed in the refrigerator. SI-NW FET was in the specified water vapor for $2 \mathrm{~h}$, at $\mathrm{T}=4{ }^{\circ} \mathrm{C}$. The water fill level did not exceed the surface height of the SI-NW FET. The antibodies were chemically bound to APTES and held on the surface. 
5. After $2 \mathrm{~h}$, antibodies were removed from the surface of the SI-NW FET that did not bind to it. The surface was washed with distilled water using a pipette. $\mathrm{V}=10 \mu \mathrm{L}$ of distilled water was applied and removed 20-30 times on the surface of the SI-NW FET. The procedure was repeated 3 times. Next, the surface of the SI-NW FET was dried. Drying was carried out in closed Petri dishes.

6. First part, experimental SI-NW FET as the biosensor is ready for used.

Sample preparation of the second part of SI-NW FET surface differed from the first only in that antibodies were not applied to the surface of the second SI-NW FET.

After completion of all sample preparation procedures, specific antibodies to CVP were found on the surface of the first part of the biosensor, and APTES was found on the surface of the second part.

Using atomic force microscopy (AFM) at the stages of sample preparation, the surface of the SI-NW FET was monitored, Figure 2a,b. Chemical modification and functionalization of the nanowire by antibodies to virus-like coronavirus particles is shown Figure 3. 

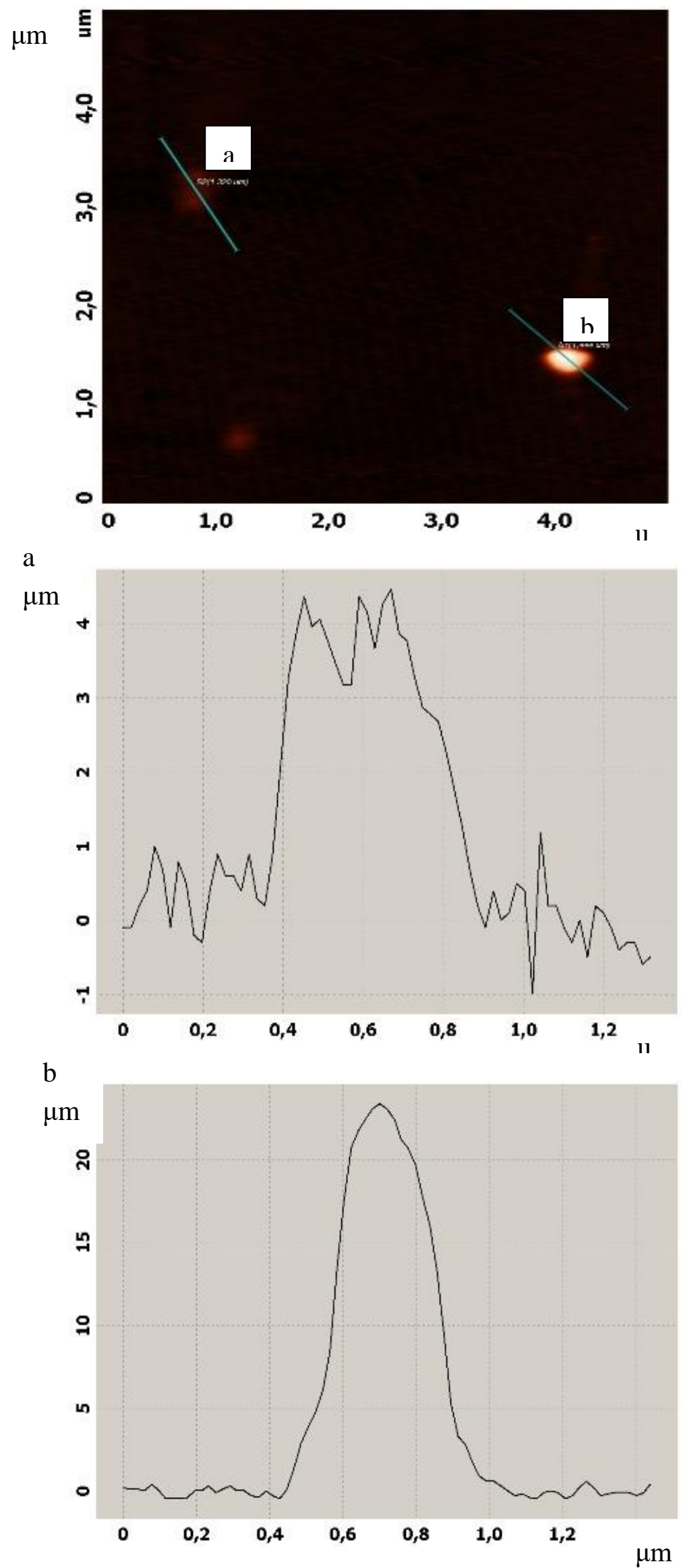

Figure 2. Antibodies on the surface of the nanowire. Atomic force microscopy. 


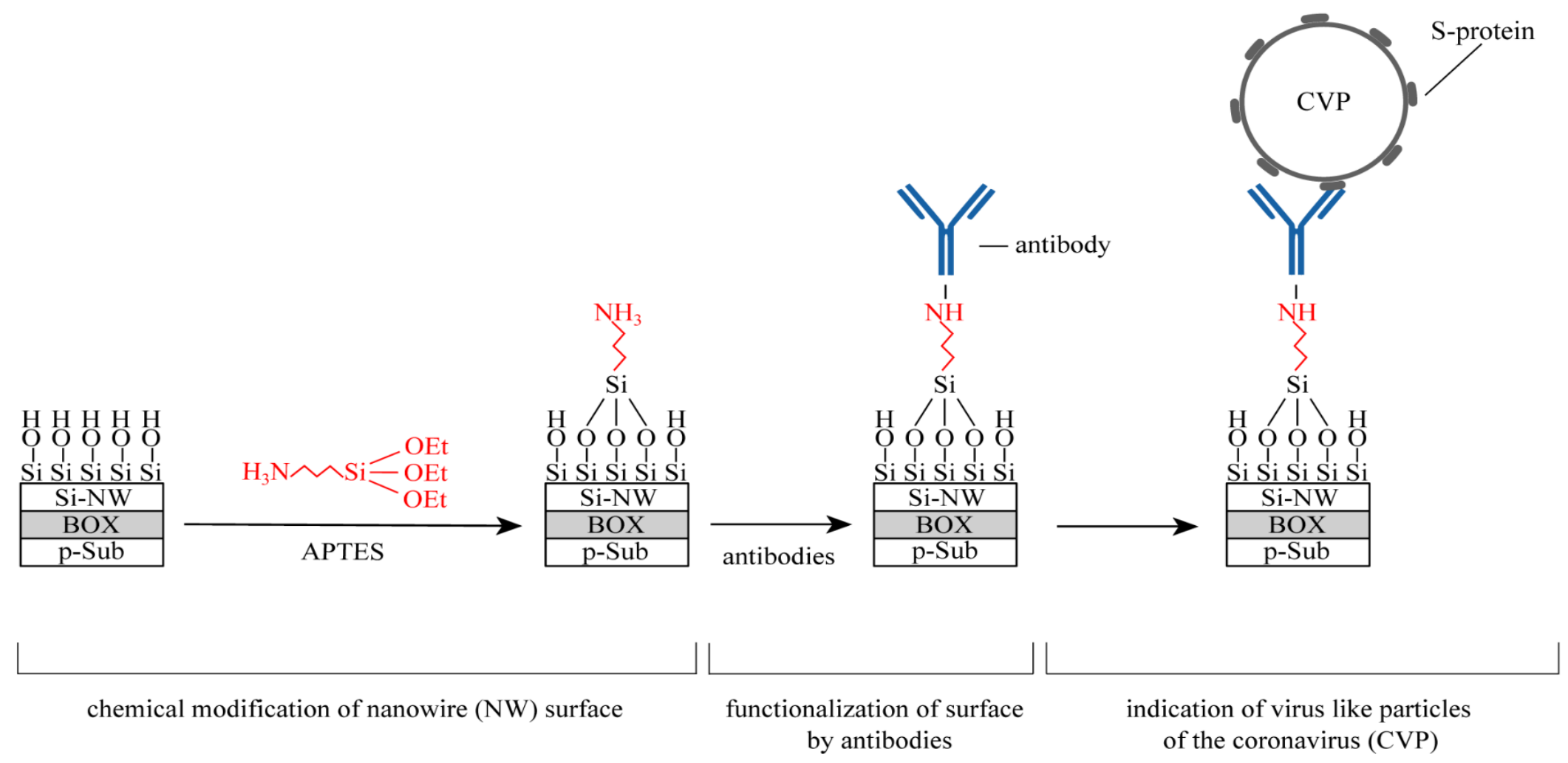

Figure 3. Chemical modification and functionalization of the nanowire by antibodies to virus-like coronavirus particles. 
During the experiment, a 1-mL viral suspension of CVP was applied to the surface of the biosensor by a pipette, so that it covered the experimental and control parts transistor (modified with antibodies and APTES).

Indication of the CVP by biosensor was carried out by measuring the current in the source-drain circuit of the experimental and control transistors in real time. The control was performed using the NI 6363 data collection system (National Instrument, USA). Before starting to register the current, the working point of both SI-NW FET transistors was set in the linear operating mode area of the voltage-ampere characteristic of the transistor. This mode provided a linear dependence of the current value of the transistor on the tested quantity of CVP.

\section{Results}

The reaction of two transistors to the introduction of a viral suspension on the surface of the same biosensor is shown Figures 4 and 5. The Red and black line currents of two independent transistors of the CNI NP in real time. The red line is a transistor reaction in response to the interaction of a specific antibody with an antigen. The black line is the control-response of the transistor to the presence of the virus, but no antibodies. The reaction of transistors in response to the introduction of a viral suspension in the amount of $10^{-13} \mathrm{M}$ was studied especially carefully Figure 3 . The process of current measurement was repeated four times.

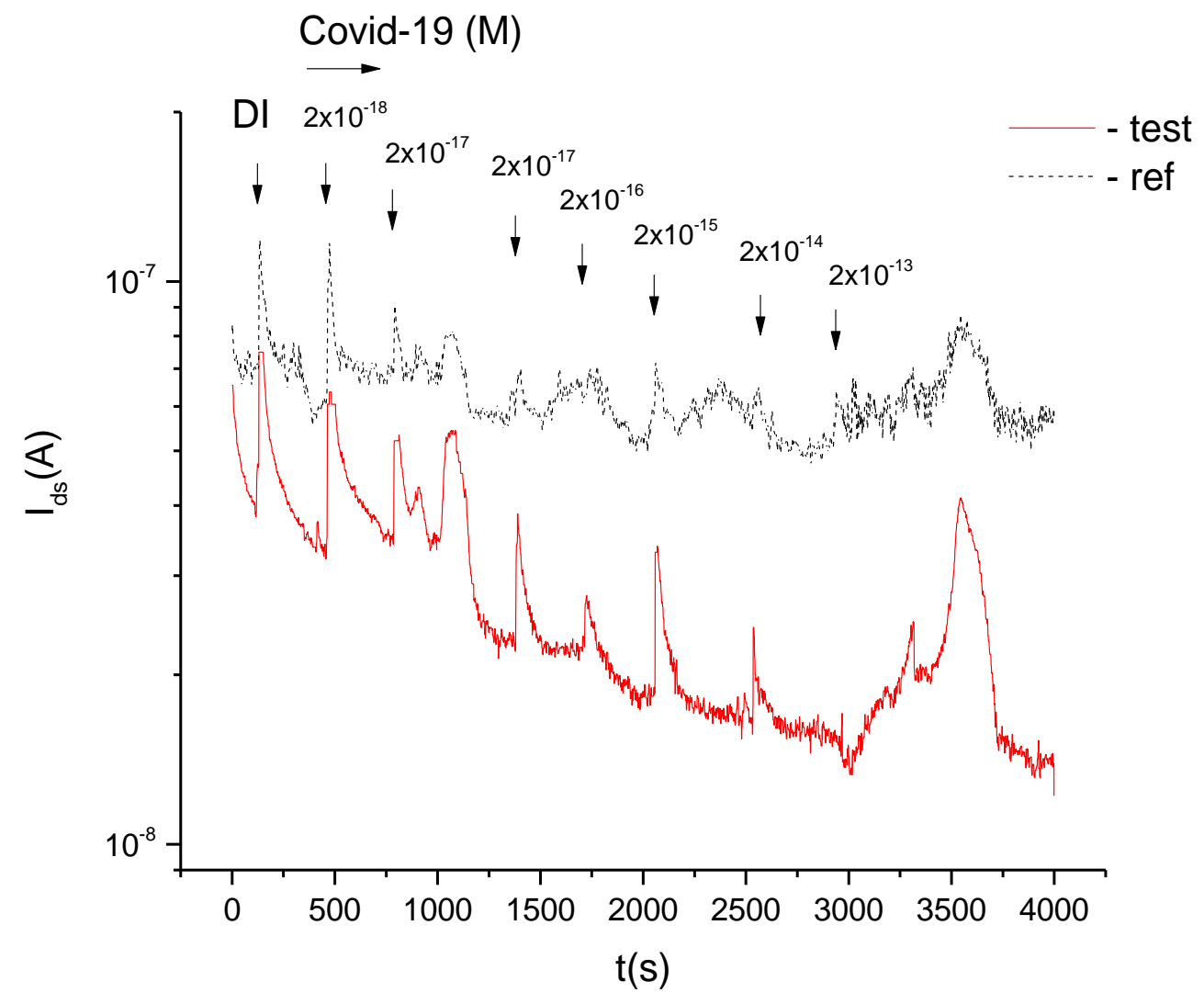

Figure 4. Experimental and control time curves of Ids $(t)$ current of two independent biosensor

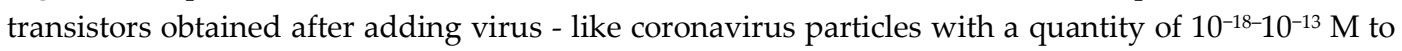
its surface. Red line (experiment) changes in the first current of a transistor in response to a specific antibody-antigen reaction on the surface of its nanowire. Black line (control) of current changes in response to the probable presence of virus-like coronavirus particles on the surface of the second transistor nanowire. 


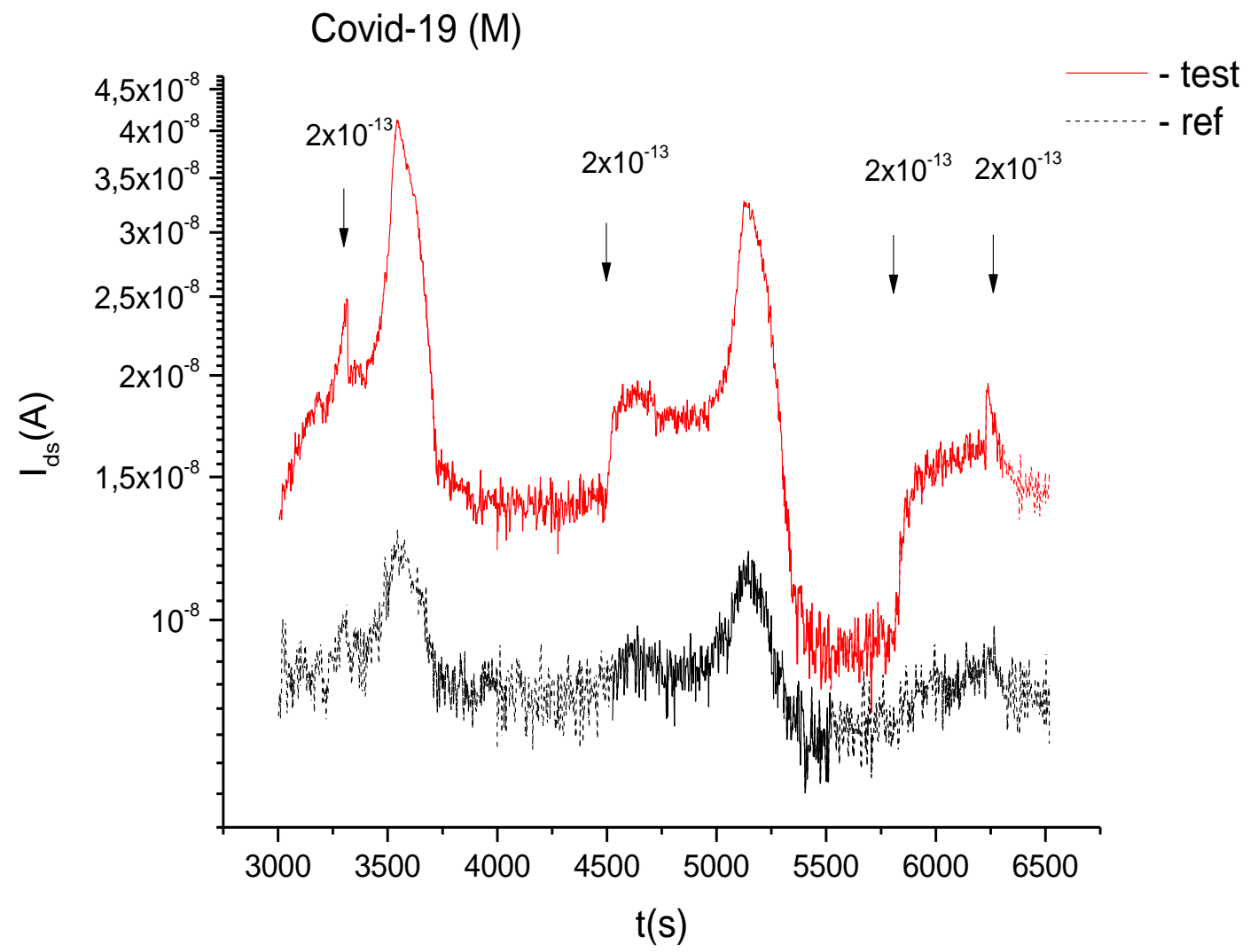

Figure 5. Experimental (red line) and control (black line) time curves of Ids ( $\mathrm{t}$ ) current of two independent biosensor transistors obtained after adding virus-like coronavirus particles with an excess quantity of $10^{-13} \mathrm{M}$ to the biosensor surface. The red line changes the first current of the transistor in response to a specific antibody-antigen reaction on the surface of its nanowire. A black line of current changes in response to the probable presence of virus-like coronavirus particles on the surface of the second transistor nanowire.

Analysis of the dynamics of changes in transistor currents Figures 4 and 5 allowed us to draw conclusions:

- $\quad$ antibody + CVP complexes, taking into account specific proteins in the suspension at the phase section with the surface of the nanowire, modulate the current of the biosensor field-effect transistor;

- HPVCS have an electrically positive charge at the phase section " nanowire surface viral suspension»;

- $\quad$ antibody + HPV complexes have an electrically negative charge on the phase section " surface of the nanowire viral suspension»;

- $\quad$ the sensitivity of the biosensor is made up of $10^{-18} \mathrm{M}$;

- $\quad$ the display time was 200-300 s.

\section{Discussion}

The experimental results are consistent with previously published results by other authors in terms of electric charge detection, indication of pathogens, proteins, oligonucleotides, etc. [8,10-12]. The results have their own unique total cost-value of electric charges. The difference of signs (positive, negative) between the electric charges for CVP, antibodies, and their antibody + antigen complexes is also noteworthy. It can be assumed that the sign of the electric charge, for example, HPV affects its interaction with the cell membrane. The basis for the assumption is the well-known 
classical interaction of positive and negative electric charges with each other. Probably, such data are of interest in the field of immunology, molecular biology, and Virology.

Enzyme-linked immunosorbent assay is based on a specific antibody-antigen relationship [7].

Specific antibodies bind to antigens (for example, CVP) due to the sum of intermolecular interactions. The energy of such a bond is high and amounts to tens of $\mathrm{kcal} / \mathrm{mol}$, so these antibody-antigen complexes are stable. The non-specific interaction of HPV also has the ability to bind to HPV, but through weak van der Waals intermolecular bonds with an energy less than one $\mathrm{kcal} / \mathrm{mol}$. Such complexes are unstable and disintegrate. This circumstance allows us to study the fundamental features of specific or non-specific interaction for specific pairs (virus + antibody), by measuring and monitoring the dynamics of the biosensor current. The use of the biosensor has prospects in the development of the technological process of vaccine production, evaluation of the quality of the material used (antigen, antibodies).

An excess of $10^{-13} \mathrm{M}$ of the virus in the suspension leads to an increase of the Debye screening length on nanowire biosensors. However, an increase in the biosensor transistor current was

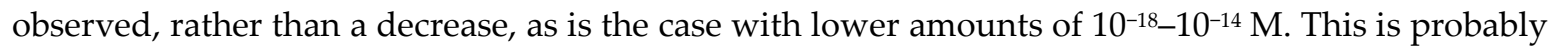
the result of electric recharge of the nanowire surface by a set of positive charges of the CVP [13].

Funding: This research was funded by State project GZ 11/16 of the industry research program of Rospotrebnadzor for 2016-2020. "Problem-oriented research in the field of epidemiological surveillance of infectious and parasitic diseases". Manufacturing of sensors and the indication process was carried out in the Rzhanov Institute of Semiconductor Physics, Siberian Branch of the Russian Academy of Sciences, Novosibirsk, Russian Federation and the support of the RFBR project No. 18-29-02091.

Author's Contribution: V.G., O.N., E.Z., B.Z., A.C. - methodology; research. V.G., O.N., D.S., S.A., A.S., B.G. - wrote and edited the article. A.A. - research conceptualization and editing. O.N., D.S., Y.M., E.Z., A.A. - resources and validation. G.B.- preparation of the original, writing and editing.

Acknowledgments: The authors thank the administration of the A. Rzhanov Institute of Semiconductor Physics, Siberian Branch of the Russian Academy of Sciences, Novosibirsk for technical support in the process of research.

Conflicts of Interest: Sponsors played no role in the development of the study; in the collection, analysis, or interpretation of data, in the writing of the manuscript, or in the decision to publish the results. All authors read and approved the final manuscript.

\section{References}

1. Coronavirus Disease (COVID-19) Pandemic. Available online: https://www.who.int/ru/emergencies/diseases/novel-coronavirus-2019

2. WHO Director-General's Opening Remarks at the Media Briefing on COVID-1919 11 March 2020 г. Available online: https://www.who.int/ru/dg/speeches/detail/who-director-general-s-opening-remarks-at-the-media-briefi ng-on-covid-19---11-march-2020

3. Coronavirus. Available online: https://www.who.int/ru/health-topics/coronavirus/coronavirus

4. Surveillance Strategies for COVID-19 Human Infection: Interim Guidance, 10 May 2020. Available online: https://apps.who.int/iris/bitstream/handle/10665/332051/WHO-2019-nCoV-National_Surveillance-2020.1rus.pdf

5. Chen, N.; Zhou, M.; Dong, X.; Qu, J.; Gong, F.; Han, Y.; Qiu, Y.; Wang, J.; Liu, Y.; Wei, Y.; et al. Epidemiological and Clinical Characteristics of 99 Cases of 2019-Novel Coronavirus (2019-nCoV) Pneumonia in Wuhan, China. Lancet 2020, 395, 507-513, doi:10.1016/S0140-6736(20)30211-7.

6. Rospotrebnadzor Research Center Has Started Testing a Vaccine Against a New Coronavirus. Available online: http://www.vector.nsc.ru/news/571/

7. Ancelevich, L.M.; Yagudin, L.A. Practical application of enzyme immunoassay in the diagnosis of diseases. Pract. Med. 2014, 3, 28-34. (In Russia)

8. Naumova, O.V.; Fomin, B.I.; Nasimov, D.A.; Dudchenko, N.V.; Devyatova, S.F.; Zhanaev, E.D.; Popov, V.P.; Latyshev, A.V.; Aseev, A.L.; Ivanov, Y.D.; Archakov, A.I. SOI nanowires as sensors for charge detection. Semicond. Sci. Technol. 2010, 25, 055004, doi:10.1088/0268-1242/25/5/055004. 
9. Prabakaran, P.; Gan, J.; Feng, Y.; Zhu, Z.; Choudhry, V.; Xiao, X.; Ji, X.; Dimitrov, D.S. Structure of severe acute respiratory syndrome coronavirus receptor-binding domain complexed with neutralizing antibody. J. Biol. Chem. 2006, 281, 15829-15836, doi:10.1074/jbc.M600697200.

10. Parka, J.; Nguyena, H.H.; Woubitc, A.; Kima, M. Applications of field-effect transistor (FET)-type biosensors. Appl. Sci. Converg. Technol. 2014, 23, 61-71, doi:10.5757/ASCT.2014.23.2.61.

11. Yang, F.; Zhang, G.-J. Silicon nanowire-transistor biosensor for study of molecule-molecule interactions. Rev. Anal. Chem. 2014, 33, 95-10, doi:10.5757/ASCT.2014.23.2.61.

12. Dmitrienko, E.; Naumova, O.; Fomin, B.; Kupryushkin, M.; Volkova, A.; Amirkhanov, N.; Semenov, D.; Pyshnaya, I.; Pyshnyi, D. Surface modification of SOI-FET sensors for label-free and specific detection of short RNA analyte. Nanomedicine 2016, 11, 2073-2082, doi:10.2217/nnm-2016-0071.

13. Stern, E.; Wagner, R.; Sigworth, F.J.; Breaker, R.; Fahmy, T.M.; Reed, M.A. Importance of the Debye screening length on nanowire field effect transistor sensors. Nano Lett. 2007, 7, 3405-3409, doi:10.1021/n1071792z.

Publisher's Note: MDPI stays neutral with regard to jurisdictional claims in published maps and institutional affiliations.

(C) 2020 by the authors. Submitted for possible open access publication under the terms and conditions of the Creative Commons Attribution (CC BY) license (http://creativecommons.org/licenses/by/4.0/). 